\title{
Mexico Transport Cost Indicator Report
}

CONTENTS
Summary: What Happened? 1
Quarterly Bulk Grain
and Soybeans
Fruit and Vegetable
Subscription Information 14
Related Websites
Data Sets

\section{Grain Transportation Costs to Mexico in First Quarter 2020}

Mexico is one of the largest importers of U.S. grain (corn, soybeans, and wheat). In 2019, Mexico imported 14.42 million metric tons (mmt) of U.S. corn, with a total value of $\$ 2.72$ billion. Also, in 2019, Mexico imported $3.55 \mathrm{mmt}$ tons of wheat (valued at $\$ 0.81$ billion) and $5.1 \mathrm{mmt}$ tons of soybeans (\$1.87 billion), according to USDA's Global Agricultural Trade System data. Because of Mexico's role as a major, nearby destination for U.S. grain, sustaining strong export levels depends heavily on retaining low transportation and landed costs. Grain is usually transported from the United States to Mexico by one of two routes-either by cross-border land movements or by seaborne movements to Mexican ports for inland distribution. This report examines changing costs of transporting grain from the United States to Mexico through the land and water routes - from fourth quarter 2019 to first quarter 2020 (quarter to quarter) and from first quarter 2019 to first quarter 2020 (year to year).

Transportation costs. Via water routes, total transportation costs of shipping grain from the United States to Mexico declined quarter to quarter. ${ }^{1}$ For corn and soybeans, this decline responded to falling truck, barge, and ocean freight rates (see table 1). For wheat, the decline was mainly in response to falling ocean freight rates. Barge rates fell because of flagging demand for barge services, with less grain shipped downriver (fig. 9 of May 14, 2020 Grain Transportation Report (GTR)). Ocean freight rates fell quarter to quarter because of the slump in the global dry bulk trade (April 16, 2020 GTR). Year to year, total transportation costs decreased for waterborne corn and soybeans and increased slightly for waterborne wheat.

Next, via land routes, total transportation costs of shipping grain from the United States to Mexico changed little from quarter to quarter. However, year to year, total transportation costs increased for all grains because of increases in truck and rail (public tariff) rates over a year ago. Rail rates held fairly steady during the quarter.

\footnotetext{
${ }^{1}$ Water routes typically involve truck transportation to barge to oceangoing vessel, or truck to rail to oceangoing vessel.
} 


\section{Mexico Transport Cost Indicator Report}

Landed costs. ${ }^{2}$ Quarter to quarter, landed costs decreased for corn and soybeans shipped to Mexico via water, but increased for waterborne wheat. Landed costs for waterborne corn and soybeans declined because of lower transportation costs and farm values. Landed costs for waterborne wheat rose mainly because of increased farm values.

For grains shipped via land, landed costs were stable for corn and soybeans quarter to quarter, while increasing for wheat. Just as for waterborne wheat, landed costs for land-hauled wheat rose with increased farm value. Year to year, landed costs decreased for all grains (combined) shipped by water routes and for wheat transported by land routes. However, landed costs increased year to year for corn and soybeans transported by land because of increased total transportation costs and farm values.

First-quarter landed costs for waterborne grains ranged from $\$ 178$ per metric ton $(\mathrm{mt})$ to $\$ 365$ per mt (see table 1 and fig. 1). For land-hauled grains, landed costs ranged from $\$ 247$ per $\mathrm{mt}$ to $\$ 411$ per $\mathrm{mt}$ (see table 1 and fig. 2). The share of landed costs for transportation ranged from 11 percent to 28 percent for the water route and from 25 percent to 41 percent for the land route (see table). In general, quarter to quarter, the transportation share of the landed cost decreased for waterborne shipments.

U.S. Export to Mexico: According to USDA's Federal Grain Inspection Service data, Mexico imported 3.13 million metric tons (mmt) of U.S. corn, $1.12 \mathrm{mmt}$ of U.S. soybeans, and $0.76 \mathrm{mmt}$ of U.S. wheat in the first quarter of 2020. Quarter to quarter, these imports amounted to 2 percent more corn, but 14 percent less wheat and 12 percent less soybeans. However, year to year, U.S. inspections for export to Mexico rose 2 percent for corn and 8 percent for wheat, while soybean inspections fell just 1 percent. Lower U.S. transportation and landed costs could keep U.S. grain shipments to Mexico competitive.

Ocean Freight Rates: Ocean freight rates for shipping bulk grains to Mexico increased during the first quarter, compared to the previous quarter, a year earlier, and the 4-year average. During the quarter, the cost of shipping a metric ton of grain, via 25,000 ton-capacity vessels from the U.S. Gulf to Veracruz, Mexico, averaged \$16.37 per mt. This is 9 percent less than the previous quarter, relatively unchanged from the same period last year, and 8 percent more than the prior 4-year average. The cost of shipping in a 35,000-40,000 ton-capacity vessel averaged $\$ 13.64$ per $\mathrm{mt}$. This represents a 10-percent decrease from the previous quarter, 2 percent decrease from the same quarter last year, and 4 percent increase from the prior 4-year average. Weak global dry bulk trade pushed down the rates for shipping bulk commodities, including grain in the third quarter (see April 16, 2020 GTR).

Railroad: In first quarter 2020, railroads transported 34,099 carloads of grain and oilseeds to Mexico-down 18 percent quarter to quarter, but up 5 percent year to year. Tariff rail rates per grain car averaged $\$ 7,701$, unchanged quarter to quarter, up 2 percent year to year, and up 4 percent from the prior 3-year average. Fuel surcharges per railcar averaged \$244, up 2 percent quarter to quarter, up 22 percent year to year, and up 62 percent from the prior 3-year average. Overall, rail transportation costs (tariff rates plus fuel surcharges) were unchanged quarter to quarter, up 3 percent year to year, and up 5 percent from the prior 3-year average.

\footnotetext{
${ }^{2}$ Landed costs include the cost of the good (farm value) and the cost to receive it (transportation costs).
} 


\section{Fruit and Vegetables}

During the first quarter of 2020, total reported shipments of fruits and vegetables from Mexico were 3.04 million tons, an 8-percent increase year to year. The sum of the top five commodities increased 100,000 tons, or 9 percent. Avocados were the largest shipment to the United States, with 294,000 tons, a 3-percent increase year to year.

Truck rates for shipments between 501 miles and 1,500 miles from the Arizona border crossings averaged \$2.53 per mile, up 15 percent from last quarter, and 1 percent higher than the same quarter last year. Rates for shipments between 501 miles and 1,500 miles from the Texas border crossings averaged \$2.49 per mile, up 12 percent from the previous quarter, and 2 percent higher than the same quarter last year.

Diesel fuel prices for border crossings through Texas averaged $\$ 2.65$ per gallon for the quarter. Diesel fuel prices for border crossings through Arizona averaged $\$ 3.08$ per gallon. Truck availability through both Arizona and Texas border crossings were reported adequate throughout the quarter. 


\section{Mexico Transport Cost Indicator Report}

\section{Table 1. Quarterly costs of transporting U.S. grain and soybeans to Mexico}

\begin{tabular}{|c|c|c|c|c|c|c|c|c|c|c|}
\hline & \multicolumn{10}{|c|}{2020} \\
\hline & \multicolumn{5}{|c|}{ Water route (to Veracruz) } & \multicolumn{5}{|c|}{ Land route (to Guadalajara) } \\
\hline & 1 st qutr & 2nd qtr & $3 r d$ qtr & 4 th qtr & Avg. & 1st qtr & 2nd qtr & $3 r d$ qtr & 4 th qtr & Avg. \\
\hline & \multicolumn{5}{|c|}{ US\$়/metric ton } & \multicolumn{5}{|c|}{ US\$̦/metric ton } \\
\hline & \multicolumn{10}{|c|}{ Corn } \\
\hline Origin & \multicolumn{5}{|c|}{ IL } & \multicolumn{5}{|c|}{ IA } \\
\hline Truck & 10.70 & & & & 10.70 & 4.62 & & & & 4.62 \\
\hline Rail $^{1}$ & & & & & & 96.35 & & & & 96.35 \\
\hline Barge & 15.55 & & & & 15.55 & & & & & \\
\hline Ocean $^{2}$ & 13.64 & & & & 13.64 & & & & & \\
\hline Total transportation cost & 39.89 & & & & 39.89 & 100.97 & & & & 100.97 \\
\hline Farm price ${ }^{3}$ & 138.05 & & & & 138.05 & 146.45 & & & & 146.45 \\
\hline Landed cost ${ }^{4}$ & 177.94 & & & & 177.94 & 247.42 & & & & 247.42 \\
\hline \multirow[t]{2}{*}{ Transport \% of landed cost } & 22.4 & & & & 22.4 & 40.8 & & & & 40.8 \\
\hline & \multicolumn{10}{|c|}{ Soybeans } \\
\hline Origin & \multicolumn{5}{|c|}{ IL } & \multicolumn{5}{|c|}{ NE } \\
\hline Truck & 10.70 & & & & 10.70 & 4.62 & & & & 4.62 \\
\hline Rail $^{1}$ & & & & & & 98.97 & & & & 98.97 \\
\hline Barge & 15.55 & & & & 15.55 & & & & & \\
\hline Ocean $^{2}$ & 13.64 & & & & 13.64 & & & & & \\
\hline Total transportation cost & 39.89 & & & & 39.89 & 103.59 & & & & 103.59 \\
\hline Farm price ${ }^{3}$ & 325.55 & & & & 325.55 & 307.30 & & & & 307.30 \\
\hline Landed cost ${ }^{4}$ & 365.44 & & & & 365.44 & 410.89 & & & & 410.89 \\
\hline \multirow[t]{2}{*}{ Transport \% of landed cost } & 10.9 & & & & 10.9 & 25.2 & & & & 25.2 \\
\hline & \multicolumn{10}{|c|}{ Wheat } \\
\hline Origin & \multicolumn{5}{|c|}{ KS } & \multicolumn{5}{|c|}{ KS } \\
\hline Truck & 4.62 & & & & 4.62 & 4.62 & & & & 4.62 \\
\hline Rail $^{1}$ & 43.31 & & & & 43.31 & 83.27 & & & & 83.27 \\
\hline Ocean $^{2}$ & 13.64 & & & & 13.64 & & & & & \\
\hline Total transportation cost & 61.57 & & & & 61.57 & 87.89 & & & & 87.89 \\
\hline Farm price ${ }^{3}$ & 160.81 & & & & 160.81 & 160.81 & & & & 160.81 \\
\hline Landed cost ${ }^{4}$ & 222.38 & & & & 222.38 & 248.70 & & & & 248.70 \\
\hline Transport \% of landed cost & 27.7 & & & & 27.7 & 35.3 & & & & 35.3 \\
\hline
\end{tabular}

${ }^{1}$ Rail rates include U.S. and Mexico portions of the movement. Mexico rail rates are estimated based on actual quoted market rates. BNSF and Union Pacific quoted rail tariff rates are through rates for shuttle trains. Rail rates include fuel surcharges, but do not include the cost of purchasing empty rail cars in the secondary market, which could exceed the rail tariff rate plus the fuel surcharge shown in the table.

2Source: O'Neil Commodity Consulting, Inc.

${ }^{3}$ Source: USDA/NASS

${ }^{4}$ Landed cost is total transportation cost plus the farm price. 


\section{Mexico Transport Cost Indicator Report}

Figure 1 . Water route shipment costs $(\$ / m t)$ to Veracruz, Mexico

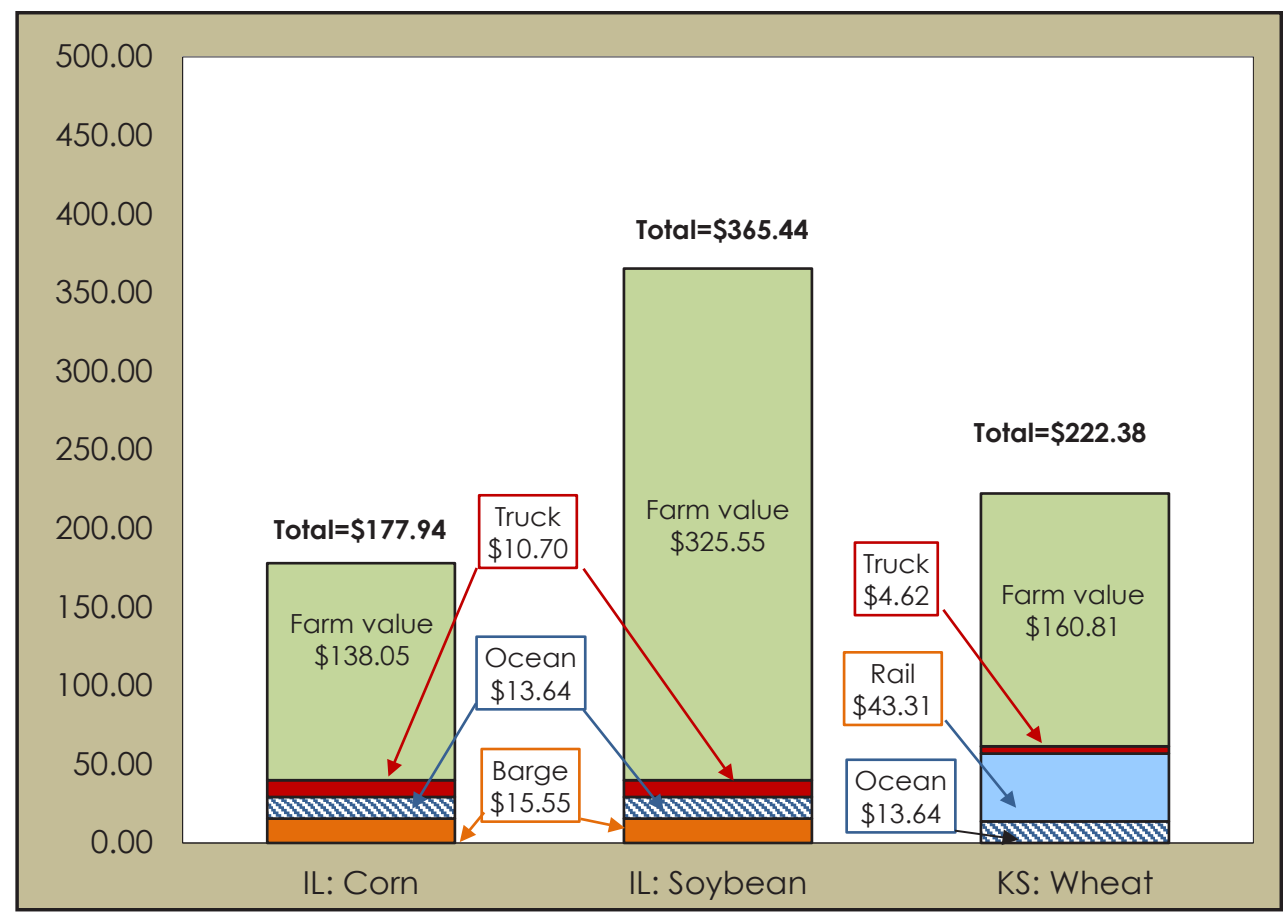

Source: USDA, Agricultural Marketing Service

Figure 2. Land route shipment costs $(\$ / \mathrm{mt})$ to Guadalajara, Mexico

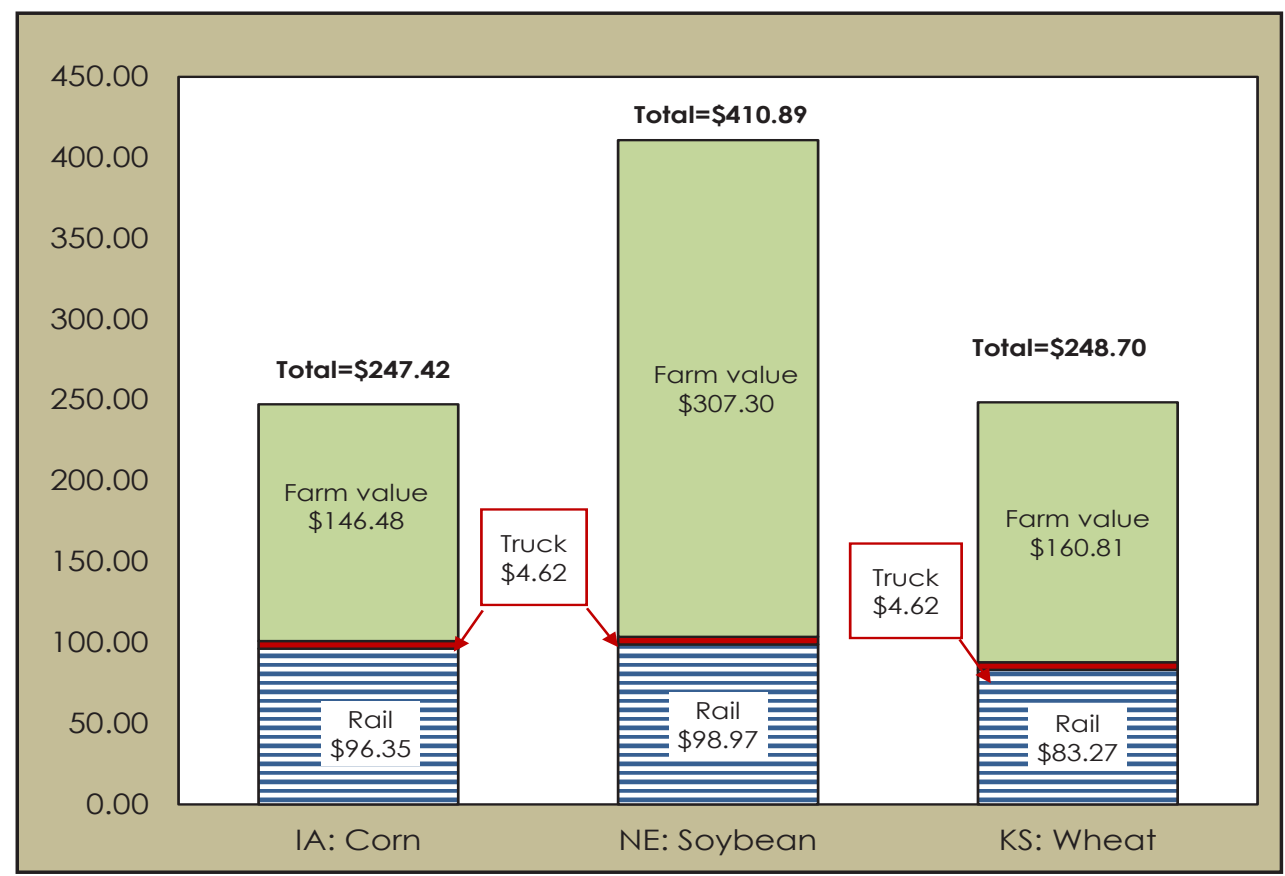

Source: USDA, Agricultural Marketing Service 


\section{Mexico Transport Cost Indicator Report}

\section{Quarterly Bulk Grain and Soybeans}

Table 2. Quarterly tariff rail rates for U.S. bulk grain shipments to Mexico (US\$/car), 2020

\begin{tabular}{|c|c|c|c|c|c|c|c|c|c|c|c|c|}
\hline \multirow[b]{2}{*}{ Commodity } & \multirow[b]{2}{*}{$\begin{array}{l}\text { Origin } \\
\text { State }\end{array}$} & \multirow[b]{2}{*}{ Destination } & \multicolumn{5}{|c|}{ Tariff rate/car ${ }^{1}$} & \multicolumn{5}{|c|}{ Fuel surcharge per car² } \\
\hline & & & $\begin{array}{l}1 \text { st } \\
\text { qtr }\end{array}$ & $\begin{array}{l}\text { 2nd } \\
\text { qtr }\end{array}$ & $\begin{array}{l}3 r d \\
\text { qtr }\end{array}$ & $\begin{array}{l}\text { 4th } \\
\text { qtr }\end{array}$ & Avg & $\begin{array}{l}1 s t \\
q t r\end{array}$ & $\begin{array}{l}\text { 2nd } \\
\text { qtr }\end{array}$ & $\begin{array}{l}3 r d \\
q t r\end{array}$ & $\begin{array}{l}\text { 4th } \\
\text { qtr }\end{array}$ & Avg \\
\hline \multirow{4}{*}{ Wheat } & MT & Chihuahua, $\mathrm{Cl}$ & 7,509 & & & & 7,509 & 0 & & & & 0 \\
\hline & OK & Cuautitlan, EM & 6,775 & & & & 6,775 & 137 & & & & 137 \\
\hline & KS & Guadalajara, JA & 7,534 & & & & 7,534 & 616 & & & & 616 \\
\hline & $\mathrm{TX}$ & Salinas Victoria, NL & 4,329 & & & & 4,329 & 83 & & & & 83 \\
\hline \multirow{6}{*}{ Corn } & IA & Guadalajara, JA & 8,902 & & & & 8,902 & 527 & & & & 527 \\
\hline & SD & Celaya, GJ & 8,140 & & & & 8,140 & 0 & & & & 0 \\
\hline & $\mathrm{NE}$ & Queretaro, QA & 8,278 & & & & 8,278 & 284 & & & & 284 \\
\hline & SD & Salinas Victoria, NL & 6,905 & & & & 6,905 & 0 & & & & 0 \\
\hline & MO & Tlalnepantla, EM & 7,643 & & & & 7,643 & 277 & & & & 277 \\
\hline & SD & Torreon, CU & 7,690 & & & & 7,690 & 0 & & & & 0 \\
\hline \multirow{4}{*}{ Soybeans } & MO & Bojay (Tula), HG & 8,547 & & & & 8,547 & 493 & & & & 493 \\
\hline & $\mathrm{NE}$ & Guadalajara, JA & 9,172 & & & & 9,172 & 515 & & & & 515 \\
\hline & IA & El Castillo, JA & 9,490 & & & & 9,490 & 0 & & & & 0 \\
\hline & KS & Torreon, CU & 7,964 & & & & 7,964 & 356 & & & & 356 \\
\hline \multirow{4}{*}{ Sorghum } & $\mathrm{NE}$ & Celaya, GJ & 7,772 & & & & 7,772 & 467 & & & & 467 \\
\hline & KS & Queretaro, QA & 8,108 & & & & 8,108 & 171 & & & & 171 \\
\hline & $\mathrm{NE}$ & Salinas Victoria, NL & 6,713 & & & & 6,713 & 137 & & & & 137 \\
\hline & NE & Torreon, CU & 7,157 & & & & 7,157 & 331 & & & & 331 \\
\hline
\end{tabular}

${ }^{1}$ Rates are based upon published tariff rates for high-capacity shuttle trains. Shuttle trains are available for qualified shipments of 75-110 cars that meet railroad efficiency requirements. The cost of obtaining empty grain cars in the Secondary Grain Car markets, which in times of high demand may exceed the tariff rate plus fuel surcharge, is not included.

${ }^{2}$ Approximate load per car $=97.87 \mathrm{mt}$ : corn \& sorghum $56 \mathrm{lbs} / \mathrm{bu}$, wheat \& soybeans $60 \mathrm{lbs} / \mathrm{bu}$ Sources: www.bnsf.com; www.uprr.com; www.kcsouthern.com 


\section{Mexico Transport Cost Indicator Report}

\section{Table 3. Quarterly tariff rail rates plus fuel surcharges for U.S. bulk grain shipments to Mexico, 2020}

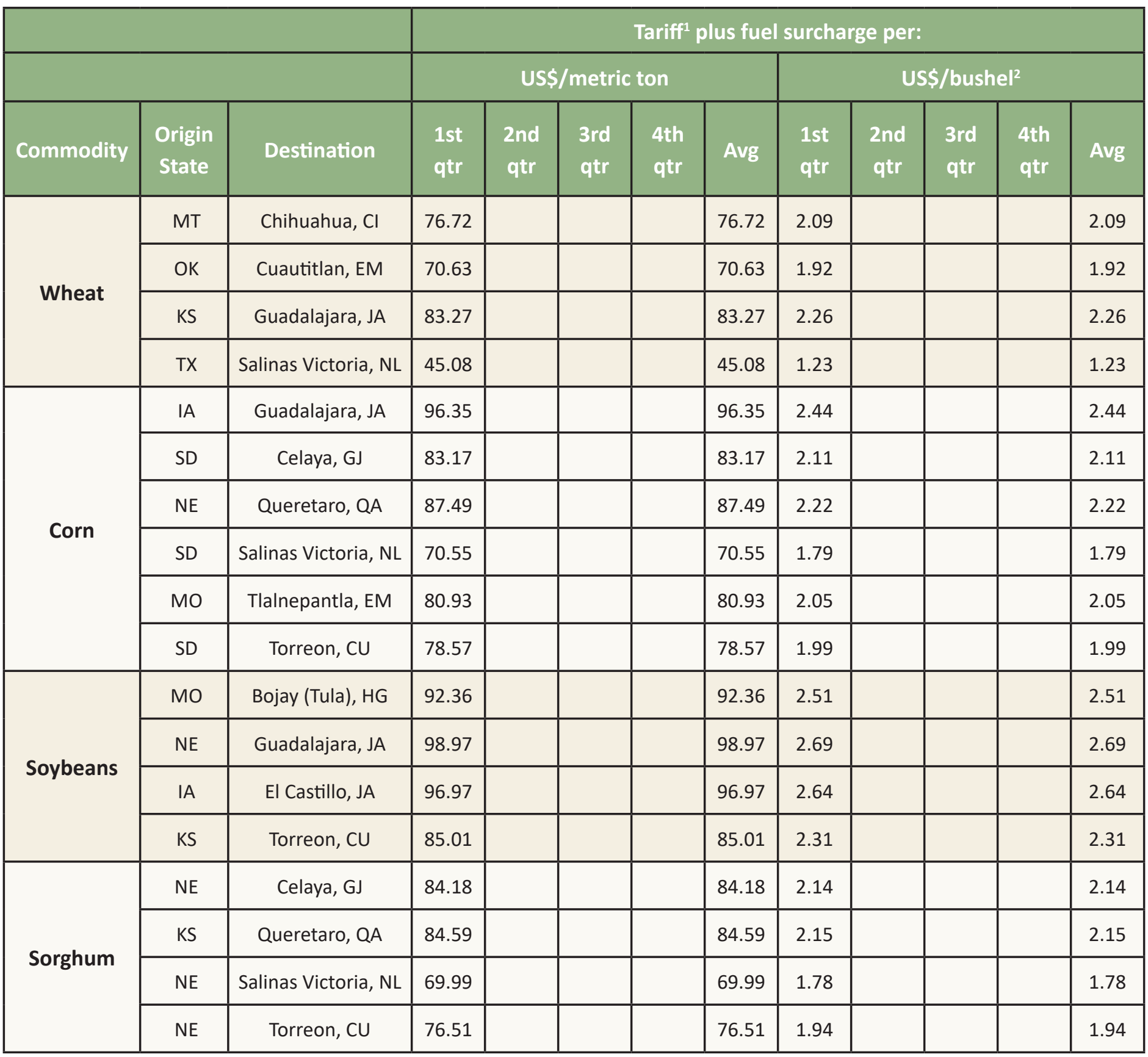

${ }^{1}$ Rates are based upon published tariff rates for high-capacity shuttle trains. Shuttle trains are available for qualified shipments of 75-110 cars that meet railroad efficiency requirements. The cost of obtaining empty grain cars in the Secondary Grain Car markets, which in times of high demand may exceed the tariff rate plus fuel surcharge, is not included.

${ }^{2}$ Approximate load per car $=97.87 \mathrm{mt}$ : corn \& sorghum $56 \mathrm{lbs} / \mathrm{bu}$, wheat \& soybeans $60 \mathrm{lbs} / \mathrm{bu}$ Sources: www.bnsf.com; www.uprr.com; www.kcsouthern.com 


\section{Mexico Transport Cost Indicator Report}

\section{Table 4. Quarterly exports of U.S. distillers' dried grains with soluble (DDGS) to Mexico*}

\begin{tabular}{|l|l|l|l|l|l|}
\hline \multirow{2}{*}{ Year } & \multicolumn{5}{|c|}{ Thousand metric tons } \\
\hline 2010 & 1 st qtr & 2nd qtr & 3rd qtr & 4th qtr & Total \\
\hline 2011 & 439 & 399 & 424 & 383 & 1,645 \\
\hline 2012 & 506 & 430 & 476 & 369 & 1,781 \\
\hline 2013 & 426 & 388 & 352 & 332 & 1,498 \\
\hline 2014 & 284 & 329 & 290 & 381 & 1,285 \\
\hline 2015 & 356 & 420 & 366 & 435 & 1,577 \\
\hline 2016 & 497 & 276 & 413 & 463 & 1,649 \\
\hline 2017 & 483 & 467 & 470 & 490 & 1,910 \\
\hline 2018 & 604 & 475 & 551 & 551 & 2,181 \\
\hline 2019 & 516 & 516 & 514 & 467 & 2,013 \\
\hline 2020 & 410 & 574 & 475 & 491 & 1,950 \\
\hline
\end{tabular}

*Data are for brewers' and distillers' dregs and waste, of which Distillers' Dried Grains with Soluble is a principal component. Source: USDA, Economic Research Service (ERS), Feed grains database 


\section{Mexico Transport Cost Indicator Report}

Table 5. Quarterly ocean freight rate for bulk grain shipments from the U.S. Gulf to Veracruz, Mexico

\begin{tabular}{|c|c|c|c|c|c|}
\hline \multicolumn{6}{|c|}{ US\$/metric ton } \\
\hline $\begin{array}{l}\text { Vessel capacity } \\
\text { (metric ton) }\end{array}$ & 1st qtr 2012 & 2nd qtr 2012 & 3rd qtr 2012 & 4th qtr 2012 & Average \\
\hline 25,000 & 20.28 & 20.79 & 20.68 & 18.73 & 20.12 \\
\hline $35-40,000$ & 18.37 & 18.62 & 18.53 & 16.73 & 18.06 \\
\hline $\begin{array}{l}\text { Vessel capacity } \\
\text { (metric ton) }\end{array}$ & 1st qtr 2013 & 2nd qtr 2013 & 3rd qtr 2013 & 4th qtr 2013 & Average \\
\hline 25,000 & 20.19 & 19.59 & 20.47 & 20.01 & 20.07 \\
\hline $35-40,000$ & 17.89 & 17.58 & 17.85 & 17.13 & 17.61 \\
\hline $\begin{array}{c}\text { Vessel capacity } \\
\text { (metric ton) }\end{array}$ & 1st qtr 2014 & 2nd qtr 2014 & 3rd qtr 2014 & 4th qtr 2014 & Average \\
\hline 25,000 & 20.08 & 17.48 & 15.75 & 16.32 & 17.41 \\
\hline $35-40,000$ & 17.53 & 15.48 & 13.56 & 13.96 & 15.13 \\
\hline $\begin{array}{l}\text { Vessel capacity } \\
\text { (metric ton) }\end{array}$ & 1st qtr 2015 & 2nd qtr 2015 & 3rd qtr 2015 & 4th qtr 2015 & Average \\
\hline 25,000 & 13.67 & 14.23 & 14.59 & 13.95 & 14.11 \\
\hline $35-40,000$ & 11.63 & 11.89 & 12.85 & 12.12 & 12.12 \\
\hline $\begin{array}{l}\text { Vessel capacity } \\
\text { (metric ton) }\end{array}$ & 1st qtr 2016 & 2nd qtr 2016 & 3rd qtr 2016 & 4th qtr 2016 & Average \\
\hline 25,000 & 12.34 & 13.47 & 15.00 & 14.85 & 13.92 \\
\hline $35-40,000$ & 10.44 & 11.65 & 13.20 & 13.26 & 12.14 \\
\hline $\begin{array}{l}\text { Vessel capacity } \\
\text { (metric ton) }\end{array}$ & 1st qtr 2017 & 2nd qtr 2017 & 3rd qtr 2017 & 4th qtr 2017 & Average \\
\hline 25,000 & 16.03 & 14.85 & 15.16 & 16.69 & 15.68 \\
\hline $35-40,000$ & 14.27 & 12.95 & 12.98 & 14.26 & 13.62 \\
\hline $\begin{array}{c}\text { Vessel capacity } \\
\text { (metric ton) }\end{array}$ & 1st qtr 2018 & 2nd qtr 2018 & 3rd qtr 2018 & 4th qtr 2018 & Average \\
\hline 25,000 & 16.11 & 16.20 & 16.68 & 17.94 & 16.73 \\
\hline $35-40,000$ & 13.97 & 14.07 & 14.68 & 15.63 & 14.59 \\
\hline $\begin{array}{c}\text { Vessel capacity } \\
\text { (metric ton) }\end{array}$ & 1st qtr 2019 & 2nd qtr 2019 & 3rd qtr 2019 & 4th qtr 2019 & Average \\
\hline 25,000 & 16.37 & 16.65 & 18.27 & 17.98 & 17.32 \\
\hline $35-40,000$ & 13.89 & 14.01 & 15.50 & 15.23 & 14.66 \\
\hline $\begin{array}{l}\text { Vessel capacity } \\
\text { (metric ton) }\end{array}$ & 1st qtr 2020 & 2nd qtr 2020 & 3rd qtr 2020 & 4th qtr 2020 & Average \\
\hline 25,000 & 16.37 & & & & 16.37 \\
\hline $35-40,000$ & 13.64 & & & & 13.64 \\
\hline
\end{tabular}

Source: O'Neil Commodity Consulting 


\section{Mexico Transport Cost Indicator Report}

FRUIT AND VEGETABLE

Table 6. Fruit and vegetable truck rates for shipments between 501 to 1,500 miles crossing the U.S.-Mexico border

\begin{tabular}{|c|c|c|c|c|c|}
\hline \multicolumn{6}{|c|}{ US\$/mile } \\
\hline Origin/border crossing & 1st qtr 2012 & 2nd qtr 2012 & 3rd qtr 2012 & 4th qtr 2012 & Average \\
\hline Nogales, Arizona & 2.00 & 2.57 & 1.84 & 1.92 & 2.08 \\
\hline Pharr, Texas & 1.97 & 2.26 & 1.89 & 2.09 & 2.05 \\
\hline Origin/border crossing & 1st qtr 2013 & 2nd qutr 2013 & 3rd qtr 2013 & 4th qtr 2013 & Average \\
\hline Nogales, Arizona & 2.34 & 2.59 & 1.63 & 2.33 & 2.22 \\
\hline Pharr, Texas & 2.15 & 2.33 & 2.02 & 2.01 & 2.13 \\
\hline Origin/border crossing & 1st qtr 2014 & 2nd qtr 2014 & 3rd qtr 2014 & 4th qtr 2014 & Average \\
\hline Nogales, Arizona & 2.46 & 2.69 & 1.74 & 2.31 & 2.30 \\
\hline Pharr, Texas & 2.32 & 2.53 & 2.12 & 2.13 & 2.28 \\
\hline Origin/border crossing & 1st qtr 2015 & 2nd qtr 2015 & 3rd qtr 2015 & 4th qtr 2015 & Average \\
\hline Nogales, Arizona & 2.41 & 2.49 & 2.71 & 2.51 & 2.53 \\
\hline Pharr, Texas & 2.26 & 2.23 & 2.50 & 2.27 & 2.32 \\
\hline Origin/border crossing & 1st qtr 2016 & 2nd qtr 2016 & 3rd qtr 2016 & 4th qtr 2016 & Average \\
\hline Nogales, Arizona & 2.31 & 2.43 & 2.53 & 2.65 & 2.48 \\
\hline Pharr, Texas & 2.98 & 2.17 & 2.24 & 2.34 & 2.43 \\
\hline Origin/border crossing & 1st qtr 2017 & 2nd qtr 2017 & 3rd qtr 2017 & 4th qtr 2017 & Average \\
\hline Nogales, Arizona & 2.05 & 2.32 & 2.45 & 2.38 & 2.30 \\
\hline Pharr, Texas & 2.16 & 2.21 & 2.00 & 2.36 & 2.18 \\
\hline Origin/border crossing & 1st qtr 2018 & 2nd qtr 2018 & 3rd qtr 2018 & 4th qtr 2018 & Average \\
\hline Nogales, Arizona & 2.92 & 3.21 & 2.75 & 2.47 & 2.84 \\
\hline Pharr, Texas & 2.95 & 3.13 & 2.27 & 2.34 & 2.67 \\
\hline Origin/border crossing & 1st qtr 2019 & 2nd qtr 2019 & 3rd qtr 2019 & 4th qtr 2019 & Average \\
\hline Nogales, Arizona & 2.52 & 2.7 & 2.52 & 2.21 & 2.49 \\
\hline Pharr, Texas & 2.45 & 2.28 & 2.04 & 2.23 & 2.25 \\
\hline Origin/border crossing & 1st qtr 2020 & 2nd qtr 2020 & 3rd qtr 2020 & 4th qtr 2020 & Average \\
\hline Nogales, Arizona & 2.53 & & & & 2.53 \\
\hline Pharr, Texas & 2.49 & & & & 2.49 \\
\hline
\end{tabular}

Source: USDA, Agricultural Marketing Service (AMS), Specialty Crops Program, Market News Division 


\section{Mexico Transport Cost Indicator Report}

\section{Table 7. Quarterly U.S.-Mexico border crossing fresh fruit and vegetables truck availability}

\begin{tabular}{|c|c|c|c|c|c|c|c|c|c|c|c|c|c|c|}
\hline \multicolumn{15}{|c|}{ 1st quarter 2020} \\
\hline Legend: & 1 =Surplus & \multicolumn{3}{|c|}{2 = Slight surplus } & \multicolumn{3}{|c|}{3 = Adequate } & \multicolumn{4}{|c|}{4 = Slight shortage } & \multicolumn{3}{|c|}{5 = Shortage } \\
\hline \multicolumn{15}{|c|}{ Truck availability } \\
\hline \multicolumn{2}{|c|}{ Mexico border crossings/month } & \multicolumn{4}{|c|}{ January } & \multicolumn{4}{|c|}{ February } & \multicolumn{5}{|c|}{ March } \\
\hline \multicolumn{2}{|c|}{ Week ending } & $1 / 7$ & $1 / 14$ & $1 / 21$ & $1 / 28$ & $2 / 4$ & $2 / 11$ & $2 / 18$ & $2 / 25$ & $3 / 3$ & $3 / 10$ & $3 / 17$ & $3 / 24$ & $3 / 31$ \\
\hline $\begin{array}{l}\text { Through } \\
\text { Nogales, AZ }\end{array}$ & $\begin{array}{c}\text { Tomatoes, Squash, } \\
\text { Cucumbers, } \\
\text { Mangoes, Honeydew, } \\
\text { Watermelons, Mixed } \\
\text { Fruits, Vegetables, } \\
\text { Mango }\end{array}$ & 4 & 3 & 3 & 3 & 3 & 3 & 4 & 4 & 3 & 3 & 4 & 2 & 1 \\
\hline Through TX & $\begin{array}{c}\text { Vegetables, Limes, } \\
\text { Mangoes, Onions, } \\
\text { Tomatoes, Broccoli, } \\
\text { Mixed Fruits }\end{array}$ & 3 & 3 & 3 & 3 & 3 & 3 & 3 & 3 & 3 & 4 & 4 & 3 & 2 \\
\hline
\end{tabular}

Source: USDA, Agricultural Marketing Service (AMS), Specialty Crop Program, Market News Division, Fruit and Vegetable Truck Rate Report

Table 8. Top ten commodities shipped by truck to the U.S. from Mexico, 2020 (10,000 lbs)

\begin{tabular}{|l|c|c|}
\hline \multicolumn{1}{|c|}{ Commodity } & 1st qtr 2020 & Rank \\
\hline Avocados & 294 & 2 \\
\hline Cucumbers & 255 & 3 \\
\hline Tomatoes & 249 & 4 \\
\hline Peppers, bell type & 243 & 5 \\
\hline Tomatoes, plum type & 231 & 6 \\
\hline Squash & 166 & 7 \\
\hline Limes & 154 & 8 \\
\hline Peppers, other & 121 & 9 \\
\hline Broccoli & 102 & 10 \\
\hline Strawberries & 98 & 2 \\
\hline
\end{tabular}




\section{Mexico Transport Cost Indicator Report}

Table 9. Top five commodities shipped by truck to the U.S. from Mexico (10,000 lbs)

\begin{tabular}{|c|c|c|c|c|c|}
\hline Commodity & 1st qtr 2013 & 2nd qtr 2013 & 3rd qtr 2013 & 4th qtr 2013 & Total 2013 \\
\hline Tomatoes (all varieties) & 88,753 & 75,505 & 43,373 & 52,154 & 259,785 \\
\hline Peppers (all varieties) & 55,952 & 35,111 & 27,341 & 51,481 & 169,885 \\
\hline Avocados & 38,933 & 26,387 & 15,049 & 30,766 & 111,135 \\
\hline Cucumbers & 38,877 & 30,555 & 11,592 & 31,523 & 112,547 \\
\hline Onions (dry and green) & 24,818 & 22,138 & 7,584 & 8,070 & 62,610 \\
\hline Subtotal & 247,333 & 189,696 & 104,939 & 173,994 & 715,962 \\
\hline Other & 206,944 & 271,688 & 126,051 & 168,680 & 773,363 \\
\hline Total & 454,277 & 461,384 & 230,990 & 342,674 & $1,489,325$ \\
\hline Commodity & 1st qtr 2014 & 2nd qutr 2014 & 3rd qtr 2014 & 4th qtr 2014 & Total 2014 \\
\hline Tomatoes (all varieties) & 102,223 & 75,885 & 41,364 & 59,367 & 278,839 \\
\hline Peppers (all varieties) & 61,170 & 32,403 & 28,315 & 49,764 & 171,652 \\
\hline Cucumbers & 25,327 & 8,7584 & 3,815 & 20,131 & 136,857 \\
\hline Avocados & 37,704 & 25,948 & 26,937 & 39,197 & 129,786 \\
\hline Squash & 4,7115 & 30,353 & 12,534 & 37,227 & 127,229 \\
\hline Subtotal & 273,539 & 252,173 & 112,965 & 205,686 & 844,363 \\
\hline Other & 218,822 & 231,589 & 126,002 & 166,317 & 742,730 \\
\hline Total & 492,361 & 483,762 & 238,967 & 372,003 & $1,587,093$ \\
\hline Commodity & 1st qtr 2015 & 2nd qtr 2015 & 3rd qtr 2015 & 4th qtr 2015 & Total 2015 \\
\hline Tomatoes (all varieties) & 97,953 & 71,449 & 45,992 & 65,381 & 280,775 \\
\hline Peppers (all varieties) & 44,215 & 37,154 & 43,044 & 49,722 & 174,135 \\
\hline Cucumbers & 59,876 & 33,752 & 30,679 & 47,396 & 171,703 \\
\hline Avocados & 23,537 & 95,273 & 7,213 & 23,195 & 149,218 \\
\hline Squash & 49,684 & 33,603 & 15,717 & 37,875 & 136,879 \\
\hline Subtotal & 275,265 & 271,231 & 142,645 & 223,569 & 912,710 \\
\hline Other & 232,251 & 250,443 & 138,828 & 185,012 & 806,534 \\
\hline Total & 507,516 & 521,674 & 281,473 & 408,581 & $1,719,244$ \\
\hline Commodity & 1st qtr 2016 & 2nd qtr 2016 & 3rd qtr 2016 & 4th qtr 2016 & Total 2016 \\
\hline Tomatoes (all varieties) & 131,455 & 89,313 & 51,983 & 66,534 & 339,285 \\
\hline Peppers (all varieties) & 61,450 & 40,970 & 33,631 & 65,270 & 201,321 \\
\hline Cucumbers & 60,241 & 37,679 & 34,993 & 40,457 & 173,370 \\
\hline Avocados & 21,726 & 85,723 & 7,560 & 33,670 & 148,679 \\
\hline Squash & 48,999 & 32,842 & 14,670 & 39,803 & 136,314 \\
\hline Subtotal & 323,871 & 286,527 & 142,837 & 245,734 & 998,969 \\
\hline Other & 270,078 & 265,393 & 157,375 & 201,602 & 894,448 \\
\hline Total & 593,949 & 551,920 & 300,212 & 447,336 & $1,893,417$ \\
\hline
\end{tabular}

Source: Data is obtained from the Department of Homeland Security (DHS), U.S. Customs and Border Protection (CBP) through USDA, AMS, Market News 


\section{Mexico Transport Cost Indicator Report}

\begin{tabular}{|c|c|c|c|c|c|}
\hline Commodity & 1st qtr 2017 & 2nd qtr 2017 & 3rd qtr 2017 & 4th qtr 2017 & Total 2017 \\
\hline Tomatoes (all varieties) & 107,852 & 82,194 & 49,088 & 73,166 & 312,300 \\
\hline Peppers (all varieties) & 67,566 & 38,714 & 31,137 & 59,172 & 196,589 \\
\hline Cucumbers & 49,565 & 36,996 & 32,133 & 47,015 & 165,709 \\
\hline Avocados & 47,336 & 32,892 & 16,064 & 44,415 & 140,707 \\
\hline Squash & 31,890 & 68,086 & 5,264 & 33,293 & 138,533 \\
\hline Subtotal & 304,209 & 258,882 & 133,686 & 257,061 & 953,838 \\
\hline Other & 291,177 & 291,747 & 170,323 & 205,516 & 958,763 \\
\hline Total & 595,386 & 550,629 & 304,009 & 462,577 & $1,912,601$ \\
\hline Commodity & 1st qtr 2018 & 2nd qtr 2018 & 3rd qtr 2018 & 4th qtr 2018 & Total 2018 \\
\hline Tomatoes (all varieties) & 105,364 & 79,851 & 49,278 & 62,478 & 296,971 \\
\hline Peppers (all varieties) & 74,252 & 46,390 & 35,103 & 57,726 & 213,471 \\
\hline Cucumbers & 55,189 & 49,914 & 35,246 & 49,781 & 190,130 \\
\hline Avocados & 51,964 & 36,452 & 14,131 & 43,288 & 145,835 \\
\hline Squash & 28,829 & 75,429 & 6,062 & 27,782 & 138,102 \\
\hline Subtotal & 315,598 & 288,036 & 139,820 & 241,055 & 984,509 \\
\hline Other & 296,266 & 281,580 & 156,781 & 205,426 & 940,053 \\
\hline Total & 611,864 & 569,616 & 296,601 & 446,481 & $1,924,562$ \\
\hline Commodity & 1st qtr2019 & 2nd qtr 2019 & 3rd qtr 2019 & 4th qtr 2019 & Total 2019 \\
\hline Tomatoes (all varieties) & 95,760 & 78,123 & 55,836 & 69,366 & 299,085 \\
\hline Peppers (all varieties) & 65,865 & 45,479 & 38,006 & 56,847 & 206,197 \\
\hline Cucumbers & 57,162 & 25,622 & 42,135 & 58,520 & 183,439 \\
\hline Avocados & 24,868 & 88,165 & 11,138 & 30,506 & 154,677 \\
\hline Squash & 48,614 & 34,729 & 18,919 & 41,334 & 143,596 \\
\hline Subtotal & 292,269 & 272,118 & 166,034 & 256,573 & 986,994 \\
\hline Other & 272,760 & 262,948 & 182,481 & 213,013 & 931,202 \\
\hline Total & 565,029 & 535,066 & 348,515 & 469,586 & $1,918,196$ \\
\hline Commodity & 1st qtr 2020 & 2nd qtr 2020 & 3rd qtr 2020 & 4th qtr 2020 & Total 2020 \\
\hline Tomatoes (all varieties) & 105,181 & & & & 105,181 \\
\hline Peppers (all varieties) & 72,764 & & & & 72,764 \\
\hline Cucumbers & 58,796 & & & & 58,796 \\
\hline Avocados & 51,075 & & & & 51,075 \\
\hline Squash & 33,236 & & & & 33,236 \\
\hline Subtotal & 32,1052 & & & & 321,052 \\
\hline Other & 287,121 & & & & 287,121 \\
\hline Total & 608,173 & & & & 608,173 \\
\hline
\end{tabular}

Source: Data is obtained from the Department of Homeland Security (DHS), U.S. Customs and Border Protection (CBP) through USDA, AMS, Market News 


\section{Mexico Transport Cost Indicator Report}

\section{Subscription Information:}

Please sign up by entering your email address at the following link and selecting your preference to receive Transportation Research and Analysis:

https://public.govdelivery.com/accounts/USDAAMS/subscriber/new?topic id=USDAAMS 177.

\section{Related Websites:}

- U.S. Grain and Soybean Exports to Mexico - A Modal Share Transportation Analysis (PDF)

- Grain Transportation Report

- Agricultural Refrigerated Truck Quarterly

\section{Data Sets (all XLS files):}

- Figure 1: Water route shipment costs $(\$ / \mathrm{mt})$ to Veracruz, Mexico

- Figure 2: Land route shipment costs $(\$ / \mathrm{mt})$ to Guadalajara, Mexico

- Table 1: Quarterly costs of transporting U.S. grain and soybeans to Mexico

- Table 2: Quarterly tariff rail rates for U.S. bulk grain shipments to Mexico (US\$/car), 2020

- Table 3: Quarterly tariff rail rates plus fuel surcharge for U.S. bulk grain shipments to Mexico, 2020

- Table 4: Quarterly exports of U.S. Distillers' Dried Grains with Soluble (DDGS) to Mexico

- Table 5: Quarterly ocean freight rate for bulk shipments from the U.S. Gulf to Veracruz, Mexico

- Table 6: Fruit and vegetable truck rates for shipments between 501 and 1,500 miles crossing the U.S.Mexico border

- Table 7: Quarterly U.S.-Mexico border crossing fresh fruit and vegetables truck availability

- Table 8: Top ten commodities shipped by truck to the U.S. from Mexico, 2019 (10,000 lbs)

- Table 9: Top five commodities shipped by truck to the U.S. from Mexico (10,000 lbs)

\section{Preferred Citation:}

U.S. Department of Agriculture, Agricultural Marketing Service. Mexico Transport Cost Indicator Reports. June 2020. Web. <http://dx.doi.org/10.9752/TS054.06-2020>

\section{Photo Credit: USDA}

USDA is an equal opportunity provider, employer, and lender.

For assistance with accessibility issues related to this document, please email sharonc.williams@usda.gov. 